Published in final edited form as:

Leuk Lymphoma. 2011 June ; 52(6): 972-985. doi:10.3109/10428194.2011.563884.

\title{
Quality of life domains among non-Hodgkin lymphoma survivors: an integrative literature review
}

\author{
ASHLEY LEAK ${ }^{1}$, DEBORAH K. MAYER ${ }^{1}$, and SOPHIA SMITH ${ }^{2}$ \\ ${ }^{1}$ School of Nursing, The University of North Carolina at Chapel Hill, NC, USA \\ ${ }^{2}$ School of Public Health, The University of North Carolina at Chapel Hill, NC, USA
}

\section{Abstract}

Survival rates of individuals with non-Hodgkin lymphoma (NHL) have increased in the past several years, as has the prevalence of older adults who are managing late and long-term effects of the disease and its treatment. In this integrative review, the state of the science for determining the quality of life (QOL) among NHL survivors is outlined. An online search of Medline, Cumulative Index to Nursing and Allied Health Literature (CINAHL), PsycINFO, and the Cochrane Library databases was conducted using the following Keywords: non-Hodgkin lymphoma, health-related quality of life, quality of life, and impact of cancer. Eighteen studies published between 2000 and 2010 are reviewed. Of these, 17 were descriptive, cross-sectional designs, and one was a systematic review. The studies included participants of varying ages and years post-diagnosis as reported in several countries. Importantly, many used one or more QOL measures as outcome variables. Future research is needed on older and minority cancer populations and should include longitudinal and interventional studies.

\section{Keywords}

Quality of life; Non-Hodgkin lymphoma; survivors; survivorship; older adult

\section{Introduction}

\begin{abstract}
Non-Hodgkin lymphoma (NHL) is the sixth most common cancer in the United States, with more than 65540 new NHL diagnoses and 20210 deaths expected in 2010 [1]. Incidence and prevalence rates increase with age, and more than $70 \%$ of NHLs are diagnosed after 55 years of age [1] and the overall 5-year survival is $68 \%$ [2]. Belonging to a group of hematologic cancers, NHL affects the white cells within the lymphatic system. Lymphoma can be slow-growing (indolent) or aggressive (fast-growing). Individuals with human immunodeficiency virus (HIV)-associated NHL are more likely to have high-grade histology and respond poorly to treatment [3]. In addition, NHL is a chronic illness with fluctuating remissions and exacerbations with varying symptoms: fevers, fatigue, weight loss, night sweats, and even localized pain dependent on the involvement of the tumors [4]. In fact, many survivors live with the disease for years and are often diagnosed during routine examinations [5].
\end{abstract}

\footnotetext{
(c) 2011 Informa UK, Ltd

Correspondence: Ashley Leak, RN-BC, MSN, OCN, School of Nursing, UNC-Chapel Hill, Carrington Hall, CB \#7460, Chapel Hill, NC 27599-7460, USA. Tel: (704)621-7751. Fax: 919-843-9900. anleak@email.unc.edu.

Potential conflict of interest: Disclosure forms provided by the authors are available with the full text of this article at www.informahealthcare.com/lal.
} 
In general, cancer is no longer synonymous with a death sentence; some types are viewed as manageable, chronic conditions, as is frequently the case with NHL. This is reflected in the change of terminology associated with cancer care; for example, individuals with cancer are no longer perceived as victims but instead as survivors who live for years following a cancer diagnosis [6,7]. While survivorship is often celebrated, cancer has a significant impact on survivors in terms of long-term health and psychosocial sequelae [6,8-11]. For example, cancer survivors are at increased risk for developing secondary malignancies and other diseases (e.g. cardiovascular disease, diabetes, osteoporosis) $[8,9,11,12]$. In addition, Hewitt et al. [13] reported that cancer survivors have an almost two-fold greater likelihood of having at least one functional limitation.

The presence of one or more coexisting conditions or ailments in addition to a primary disease such as cancer is known as a comorbidity [14,15]. When a survivor has another comorbid condition, they may experience a decline in functional status that can negatively influence their quality of life (QOL). These comorbid conditions become more prevalent as people age, complicating the illness trajectory of NHL. However, the psychosocial and QOL needs of older adults with NHL remain understudied [4]. Because few data exist on older NHL survivors and the effects of various treatments on their QOL, it is important to understand their QOL domains in order to better address their needs. The purpose of this article is to provide an integrative review that reports on the science for determining QOL among older NHL survivors. The review is focused on QOL measures and treatment effects in the identified studies.

\section{Methods}

Articles were retrieved for review via a combination of computer and manual searches of selected QOL and cancer-related publications. A comprehensive, online database search of Medline, Cumulative Index to Nursing and Allied Health Literature (CINAHL), PsycINFO, and the Cochrane Library was conducted for NHL research articles published between January 2000 and April 2010. The following search terms were used alone and in combination: non-Hodgkin lymphoma, health-related quality of life, quality of life, and impact of cancer.

Since there is a paucity of data regarding the QOL of older NHL survivors, studies that included younger NHL survivors and those with subsamples of NHL survivors were used in this analysis. However, there was a lack of clarity in age delineation in several studies, but it is assumed that individuals aged $\geq 65$ years are older adults per Medicare requirements, unless explicitly stated otherwise [16]. Except for Mols et al. [16], no studies delineated age.

Articles focused on central nervous system and T-cell cutaneous lymphomas were excluded because their treatment and clinical courses differ from other NHL subtypes [17].

Additionally, publications lacking a standardized QOL-related outcome measure were excluded. Articles were retrieved in the English language, and publication types were limited to primary research reports and systematic reviews. Editorials, opinions, and case studies were excluded.

Articles were reviewed initially by abstracts and titles, yielding 42 abstracts from Medline, 143 abstracts from CINAHL, 12 abstracts from PsycINFO, and no abstracts from the Cochrane Library. Duplicate articles were excluded, as were abstracts that did not meet the inclusion criteria. The full texts of the remaining 98 articles were read. Eighteen articles met the inclusion criteria and were selected for review. Each article was critiqued and appraised for the quality of the research evidence in relationship to QOL for NHL survivors (Figure 1). 


\section{Results of the literature review \\ General characteristics}

The majority (15/18) of the selected studies were published within the past 5 years. Twelve of the studies were based in the USA, which has a larger cancer database and registry than those in the countries of the remaining studies: The Netherlands $(n=2)$, Canada $(n=1)$, Israel $(n=1)$, and the United Kingdom $(n=1)$. Seventeen of the studies were descriptive, used a cross-sectional design with varying age ranges, and reported on the number of years post-diagnosis. Sociodemographic characteristics were homogeneous across studies.

\section{Quality of life measures}

It is generally agreed that QOL is a subjective, multidimensional construct with both positive and negative aspects [18]. QOL domains include physical, psychological, social, functional, and spiritual/existential; each will be addressed. Instruments vary, with the physical well-being domain included most often; however, no QOL instrument is accepted consistently as a gold standard [19]. As shown in Table I, half of the studies $(n=9)$ used more than one QOL outcome measure. Additional instruments that are not included in Table I are the Functional Assessment of HIV Infection [20], Medical Outcomes Study-Social Support [21], Life Impact [22], PTSD Inventory [23], and PTSD Checklist-Civilian Version [24].

Physical well-being-Multiple chronic conditions can affect functional abilities and can decrease a person's ability to maintain a healthy lifestyle. Using the Impact of Cancer (IOC) scale, Zebrack et al. [25] measured QOL in a sample of individuals with cancer (including 49 lymphoma survivors) who were 5-10 years post-diagnosis $(n=193)$. They found that older adults reported better overall QOL $(p=0.004)$ but worse physical health $(p=0.04)$ than their younger counterparts. Lower income $(p=0.02)$ and comorbidities $(p=0.003)$ were associated with worse physical functioning. A higher negative impact summary score was associated with worse physical functioning $(p<0.0001)$, worse mental health $(p<0.0001)$, and lower overall QOL $(p<0.0001)$.

These findings were consistent with the results of Smith et al. [26]. They measured NHL survivors' $(n=652)$ perceptions of positive and negative impact of cancer domains related to health status and QOL and found that survivors who were non-Caucasian, without a college degree, unemployed, younger at study enrollment, or had comorbid conditions or less social support also had lower QOL. In a related study, survivors with active NHL disease were found to have worse physical functioning and QOL than disease-free survivors [27].

While cancer-related detriments to physical functioning are common, it has been shown that healthy behaviors such as physical activity can improve QOL and reduce symptoms such as fatigue in cancer survivors [28]. In a cross-sectional study of NHL survivors $(n=438)$, differences in overall QOL were found between NHL survivors who met and did not meet recommended public health exercise guidelines of at least 150 min per week of moderate to strenuous activity [29]. The NHL survivors who met these guidelines reported higher QOL than those who did not meet them, suggesting that individuals who exercise at least $150 \mathrm{~min}$ per week receive physical and mental health benefits [30]. Bellizzi et al. [31] also explored physical activity as it related to QOL and found that survivors who engaged in some form of physical activity reported better physical and mental health $(p<0.001)$. Furthermore, Reeve et al. [32] examined QOL among older adults using the Surveillance, Epidemiology, and End Results-Medicare Health Outcomes Survey in a longitudinal, population-based study. Changes in QOL from before and after the cancer diagnoses were reported in nine cancer groups including NHL; the NHL survivors $(n=53)$ had one of the highest declines in 
physical health. In summary, these studies highlight that older adult cancer survivors and those with comorbid illnesses experience more physical health problems and worse physical QOL compared to their younger counterparts and healthy controls; however, physical activity can provide some protective effects and improve QOL in these individuals.

Psychological well-being-Psychological changes occur during the survivorship trajectory and can be stressful on the mind and body, resulting in negative health outcomes for the survivor. How an individual appraises his or her diagnosis or the perceived threat of the cancer can affect clinical and psychological outcomes [33]. The Diagnostic and statistical manual of mental disorders, 4th edition, recognizes that a cancer diagnosis meets the criteria of a traumatic stressor [34]. For example, survivors of NHL may develop symptoms of posttraumatic stress disorder (PTSD) that are also reported in individuals who experience a non-cancer-related traumatic event (e.g. motor vehicle accident, sexual assault, military combat) [23,24].

In a cross-sectional study of NHL survivors $(n=886), 8 \%$ met the criteria for a full PTSD diagnosis (compared to an estimated $2.4 \%$ prevalence in the general adult population), $9.1 \%$ met partial criteria, and 39\% of the total sample had PTSD symptoms in at least one of the three domains used for diagnosis [24]. In addition, Geffen et al. [23] found that NHL survivors who had more PTSD symptoms also experienced lower physical QOL.

In other studies, lymphoma survivors were found to have mental health functioning comparable to the general population [31]. However, survivors with active NHL disease had worse mental health functioning than those individuals in remission. In addition, younger NHL survivors (25-34 and 45-55 years of age) had lower mental summary scores than the general population [27]. In summary, it has been shown that a cancer diagnosis affects an individual's psychological well-being and their ability to cope during a time of uncertainty.

Social well-being-Social well-being encompasses the interpersonal relationships that may have been changed by the cancer experience as the survivor reintegrates into the social environment. Aside from receiving the diagnosis, the treatment for NHL can have a negative effect on various QOL domains, including changes in social relationships. Social functioning after a cancer diagnosis may be a long-term adjustment for survivors, warranting further exploration.

In one study, Bellizzi et al. [31] reported that NHL had a positive effect on relationships but a negative effect on a survivor's current financial situation. The social impact of financial and relationship changes led survivors to other sources of social support including formal and informal sources. Social support was a significant positive psychosocial variable with a mean of 83.1 (SD 16.4) on a 20-100 scale. This number indicates that many NHL survivors perceive to have supportive resources or individuals that are important to them during times of unpredictability and change [27]. Employment, financial, and insurance issues are also ongoing concerns of survivors and are addressed in the 'Functional well-being' section below.

Functional well-being-The Functional Assessment of Cancer Therapy-General (FACTG) measure includes functional well-being as one of its QOL domains. Six of the studies used the FACT-G instrument with consistent findings that one or more health problems coupled with NHL can lead to a decline in functional well-being. Chronic conditions can impede a survivors' ability to maintain healthy behaviors and lifestyles, further contributing to a decline in function. Hewitt et al. [13] reported that cancer survivors, including those of NHL, have an almost two-fold likelier chance of having at least one functional limitation. When an additional chronic condition is combined with cancer, functional limitation 
increases. In addition, Diamond et al. [20] reported that HIV-infected survivors with NHL have worse overall QOL and survival rates than uninfected survivors with NHL, indicating that having more than one life-limiting illness had a detrimental effect on the overall health and survival of their study participants.

Smith et al. [27] reported that $60 \%$ of NHL survivors were either unemployed or retired due to issues related to their cancer. In addition, how NHL survivors perceive their illness can influence their functional and physical abilities, which can impede their QOL [26].

Functional change at any juncture of the survivorship journey may require a change in living arrangements and the amount of support needed. This QOL domain continues to gain more interest as the population ages, in terms of the related impacts on daily living.

In one study, survivors who were able to maintain their work status reported higher physical QOL compared to those who reduced their work hours or stopped working completely [35]. Forty-one percent of the participants in the same study reported a change in work status and related QOL decline due to their cancer, along with problems obtaining insurance or a mortgage.

Not only do NHL survivors experience physiological effects with risks of disability and poor functioning, but also psychological stresses of healthcare expenditures on their QOL. In addition, individuals with chronic health conditions (including cancer survivors) account for the majority of US healthcare expenses. Assessment of QOL may direct interventions that reduce healthcare costs and, ultimately, improve QOL [36].

Spiritual and existential well-being-Cancer survivors' experiences parallel those of individuals who have experienced other traumatic stressors, involving acute, unpredictable events that threaten an individual's life. However, few studies have assessed the positive psychological changes or benefits from the illness [31,37]. In one of these studies, the majority of cancer survivors reported that experiencing a traumatic event, such as the diagnosis of a life-threatening illness, led to positive personal changes, a greater ability to cope with life stressors, and a sense of personal strength [33]. Posttraumatic growth is an interrelated concept that has been linked with the spiritual and existential QOL domain, or a 'perceived positive psychological change experienced as a result of the struggle with highly challenging life circumstances' [38]. Exploration of the role of spirituality has increased in recent years, but is typically not addressed or described in research studies.

In addition, having cancer often prompts individuals to revisit their outlook on life. Zebrack's study [25] used a qualitative approach with face-to-face, semistructured interviews to explore four QOL domains (physical, psychological, social, and spiritual or existential). The spiritual and existential domain of QOL was reflected in finding purpose and meaning in life, both of which were critical components in the survivorship trajectory. Zebrack et al. [25] found that having a purpose in life and hopefulness were highly rated spiritual subscale items, leading to a conclusion that survivors who are hopeful have a higher QOL.

\section{Treatment effects on QOL}

Watch-and-wait or active surveillance approaches for indolent types of NHL are the most conservative management strategies. Surveillance involves monitoring through blood tests and physical examinations until the symptoms progress or the cancer interferes with the survivor's QOL [39]. Individuals with fast-growing or aggressive NHL often present with symptoms such as fatigue, weight loss, night sweats, or complaint of sore lymph nodes [40]. Aggressive NHL is commonly treated with targeted biologic therapies such as rituximab. 
Most NHL survivors included in these studies received chemotherapy, radiation therapy, rituximab, or some combination of them for their treatment. The NHL survivors treated with chemotherapy or with active disease reported significantly worse psychological and social well-being and health-related QOL than those who had not received chemotherapy [20,38]. The survivors who reported significantly less vitality and worse general health compared with the general population [32,41], and those treated with either radiation therapy or watchful waiting, did not report a worse QOL compared with the general population [42]. Survivors who relapsed had worse QOL and physical and mental functioning compared to newly diagnosed NHL survivors [43].

Persson and Hallberg [44] explored the experience of receiving lymphoma and leukemia treatment using a phenomenological design. Three themes emerged from their research about what survivors felt while undergoing treatment: belief in life (they fought for it and came through stronger); life went on (they adapted and found a balance in the new life); or life was over (they felt out of control and lost belief in life). The impact of the disease varied depending on the survivor's illness trajectory and their ability to cope with the disease's unpredictability. This study provided meaning of the experiences felt by lymphoma and leukemia survivors and allowed them to retell their story of living with the disease.

\section{Older adults with NHL}

Age-related issues that impact QOL for NHL survivors were examined in fewer than 10 studies. For those that did, older age was associated with worse physical QOL but better mental health compared with younger adults with NHL [16,27,32,39, 41,45,46]. Most of the survivors studied received chemotherapy in combination with radiation therapy, surgery, bone marrow transplant, stem cell transplant, or biological therapies. Additionally, older survivors who received chemotherapy had poorer psychological and social well-being compared to those who did not receive chemotherapy $[16,35]$.

It is not uncommon that older adults have one or more coexisting illnesses coupled with cancer [15], which is a finding that needs to be explored. Further research within this population will provide new insights into how older NHL survivors manage their multiple comorbidities and the impact on their QOL.

\section{Design and framework}

The design and framework of a study impacts the quality of the data and how it relates to other areas of research. The methods used in the reviewed studies (Table II) had both negative and positive effects on the ability to use the data as an accurate measurement of the QOL in NHL survivors. Large, cross-sectional studies are generally less costly than longitudinal studies but are limited to describing only associations within the sample. For example, cross-sectional designs do not answer cause and effect but provide useful information in the exploration of relationships among QOL domains and the effects of cancer treatments.

The lack of a conceptual or theoretical model can be problematic when QOL is used to guide the study's design or is viewed as the outcome variable. While theoretically driven studies allow relationships to be tested in the model, only three reviewed studies identified conceptual or theoretical perspectives [24,27,45]. Inclusion of a model addressing the multidimensionality of QOL and its interrelationships or factors among QOL domains allows for hypotheses and relationships to be tested [47]. Using theoretically based models of QOL increases applicability of the concept and contributes to the ongoing development of cancer survivorship models. Ultimately, intervention studies to improve QOL in NHL survivors could be developed based on these models. 


\section{Discussion}

This article provides an integrative review of the survivorship literature relevant to older NHL survivors with a focus on the QOL measures and treatment methods used. While a limited number of studies examining QOL factors of older NHL survivors were found, the literature reflected growing interest in understanding the QOL among NHL survivors as a group. This is evidenced by recently published research (within the last decade) from around the world. Limitations of this literature review include underrepresentation of minority NHL survivors, lack of longitudinal studies and theoretical or conceptual frameworks, limited findings specific to older adults, and lack of literature regarding long-term survivors. In other cancer groups, racial and/or ethnic differences in QOL have been reported; therefore, future NHL studies with increased minority representation may find similar disparities [48]. Also, qualitative studies are needed to enhance the understanding of the impact that NHL has on QOL for survivors, particularly in minority and older populations. Finally, mixed methods studies that complement quantitative findings and explore the experiences of NHL survivors, such as Zebrack's [25] study, in which survivors shared their experiences of receiving their cancer diagnoses and their uncertainties about the future associated with decreased QOL, would be beneficial. This is one example of how quantitative and qualitative methods informed one another, at least in the spiritual and existential domain of QOL.

While QOL was measured objectively in all of the studies, it is also important to understand the subjective nature of the multidimensionality of QOL, which allows for survivors to express their experiences more thoroughly. Using qualitative methods to complement standardized QOL-related instruments would more adequately capture the totality of a survivor's experience compared with using one measure.

\section{Implication for future research}

Cancer survivors are at increased risk for QOL-related concerns due to their exposure to disease and treatment-related effects (e.g. recurrences, secondary malignancies, cardiotoxicities, social and financial issues) compared to the general population [10]. Lack of understanding of the QOL outcomes (physical, psychological, social, functional, and spiritual/existential) of older adults with cancer is a growing health concern due to the aging of the US population. For survivors of all ages, there is increasing information on intermediate ( $2-5$ years post-diagnosis) and longer-term ( $>5$ years post-diagnosis) adjustment to NHL. Increasing sociodemographic diversity in the sample could also enhance the generalizability of the findings.

As the growing population of older NHL survivors increases, research focused on QOLrelated outcomes is needed. Future studies are needed to build on descriptive, cross-sectional designs and provide a more comprehensive understanding of the survivorship journey. Longitudinal designs in cancer survivorship can help specify under what circumstances the process of cancer survivorship adaptation is best described [6]. From these longitudinal findings, interventions tailored specifically to NHL survivors will determine who responds to the intervention and the sustainable effect of the intervention. Study findings could be generalized also to a larger, long-term, survivor population.

\section{References}

1. American Cancer Society. Cancer facts \& figures [Internet]. 2010. Available from: http://www.cancer.org/downloads/STT/Cancer_Fact_and_Figures_2010.pdf

2. Horner, M.; Ries, L.; Krapcho, M., editors. SEER Cancer Statistics Review, 1975-2006 [Internet]. National Cancer Institute; Available from: http://seer.cancer.gov/csr/1975_2006 
3. Cote T, Biggar R, Rosenberg P, et al. Non-Hodgkin's lymphoma among people with AIIDS: incidence, presentation and public health burden. Int J Cancer. 1997; 73:645-650. [PubMed: 9398040]

4. National Cancer Institute. Non-Hodgkin lymphoma [Internet]. 2010. Available from: http://www.cancer.gov/cancertopics/types/non-hodgkin

5. Hamblin T. Achieving optimal outcomes in chronic lymphocytic leukaemia. Drugs. 2001; 61:593611. [PubMed: 11368285]

6. Aziz N, Rowland J. Trends and advances in cancer survivorship research: challenge and opportunity. Semin Radiat Oncol. 2003; 13:248-266. [PubMed: 12903014]

7. Bloom J. Surviving and thriving? Psychooncology. 2002; 11:89-92. [PubMed: 11921324]

8. Brown J, Byers T, Doyle C, et al. Nutrition and physical activity during and after cancer treatment: an American Cancer Society guide for informed choices. CA Cancer J Clin. 2003; 53:268-291. [PubMed: 14570227]

9. Centers for Disease Control and Prevention. Cancer survivorship-United States, 1971-2001. MMWR Morb Mortal Weekly Rep. 2004; 53:526-529.

10. Ganz P. Late effects of cancer and its treatment. Semin Oncol Nurs. 2001; 17:241-248. [PubMed: 11764707]

11. Jemal A, Clegg L, Ward E, et al. Annual report to the nation on the status of cancer, 1975-2001, with a special feature regarding survival. Cancer. 2004; 101:3-27. [PubMed: 15221985]

12. Bolin K. Health among long-term survivors of breast cancer: an analysis of 5-year survivors based on the Swedish surveys of living conditions 1979-1995 and the Swedish Cancer Registry 2000. Psychooncology. 2008; 17:1-8. [PubMed: 17385191]

13. Hewitt M, Rowland J, Yancik R. Cancer survivors in the United States: age, health, and disability. J Gerontol A Biol Sci Med Sci. 2003; 58:82-91. [PubMed: 12560417]

14. Yancik R, Ganz P, Varricchio C, et al. Perspectives on comorbidity and cancer in older patients: approaches to expand the knowledge base. J Clin Oncol. 2001; 19:1147-1151. [PubMed: 11181680]

15. Yancik R, Havlik R, Wesley M, et al. Cancer and comorbidity in older patients: a descriptive profile. Ann Epidemiol. 1996; 6:399-412. [PubMed: 8915471]

16. Mols F, Coebergh J, van de Poll-Franse L. Health-related quality of life and health care utilisation among older long-term cancer survivors: a population-based study. Eur J Cancer. 2007; 43:22112221. [PubMed: 17689955]

17. National Comprehensive Cancer Network. Non Hodgkin's lymphoma overview [Internet]. 2010. Available from: http://www.nccn.com/treatment-summaries/non-hodgkins-lymphoma.html

18. Cella D, Tulsky D, Gray G, et al. The Functional Assessment of Cancer Therapy Scale: development and validation of the general measure. J Clin Oncol. 1993; 11:570-579. [PubMed: 8445433]

19. Donnelly S. Quality-of-life assessment in advanced cancer. Curr Oncol Rep. 2000; 2:338-342. [PubMed: 11122862]

20. Diamond C, Taylor T, Anton-Culver H. Quality of life, characteristics and survival of patients with HIV and lymphoma. Qual Life Res. 2010; 19:149-155. [PubMed: 20058087]

21. Sherbourne C, Stewart A. The MOS social support survey. Soc Sci Med. 1991; 32:705-714. [PubMed: 2035047]

22. Bellizzi K, Miller M, Arora N, et al. Positive and negative life changes experienced by survivors of non-Hodgkin's lymphoma. Ann Behav Med. 2007; 34:188-199. [PubMed: 17927557]

23. Geffen D, Blaustein A, Amir M, et al. Post-traumatic stress disorder and quality of life in longterm survivors of Hodgkin's disease and non-Hodgkin's lymphoma in Israel. Leuk Lymphoma. 2003; 44:1925-1929. [PubMed: 14738144]

24. Smith S, Zimmerman S, Williams C, et al. Post-traumatic stress outcomes in non-Hodgkin's lymphoma survivors. J Clin Oncol. 2008; 26:934-941. [PubMed: 18281667]

25. Zebrack B, Yi J, Petersen L, et al. The impact of cancer and quality of life for long-term survivors. Psychooncology. 2008; 17:891-900. [PubMed: 18050153] 
26. Smith S, Crespi C, Petersen L, et al. The impact of cancer and quality of life for post-treatment non-Hodgkin lymphoma survivors. Psychooncology. 2010; 19:1259-1267. [PubMed: 20099255]

27. Smith S, Zimmerman S, Williams C, et al. Health status and quality of life among non-Hodgkin lymphoma survivors. Cancer. 2009; 115:3312-3323. [PubMed: 19452546]

28. Pinto B, Rabin C, Abdow S, et al. A pilot study on disseminating physical activity promotion among cancer survivors: a brief report. Psychooncology. 2008; 17:517-521. [PubMed: 17847122]

29. Vallance J, Courneya K, Jones L, et al. Differences in quality of life between non-Hodgkin's lymphoma survivors meeting and not meeting public health exercise guidelines. Psychooncology. 2005; 14:979-991. [PubMed: 15744764]

30. Pate R, Pratt M, Blair S, et al. Physical activity and public health: a recommendation from the Centers for Disease Control and Prevention and the American College of Sports Medicine. JAMA. 1995; 273:402-407. [PubMed: 7823386]

31. Bellizzi K, Rowland J, Arora N, et al. Physical activity and quality of life in adult survivors of nonHodgkin's lymphoma. J Clin Oncol. 2009; 27:960-966. [PubMed: 19139438]

32. Reeve B, Potosky A, Smith A, et al. Impact of cancer on health-related quality of life of older Americans. J Natl Cancer Inst. 2009; 101:860-868. [PubMed: 19509357]

33. Tedeschi, R.; Park, C.; Calhoun, L. Posttraumatic growth: conceptual issues. In: Tedeschi, R.; Park, C.; Calhoun, L., editors. Posttraumatic growth: positive changes in the aftermath of crisis. Mahwah, NJ: Erlbaum; 1998. p. 1-22.

34. American Psychiatric Association. Diagnostic and statistical manual of mental disorders. 4. Washington, DC: APA; 2000.

35. Mols F, Thong M, Vreugdenhil G, et al. Long-term cancer survivors experience work changes after diagnosis: results of a population-based study. Psychooncology. 2009; 18:1252-1260. [PubMed: 19156675]

36. Baker F, Haffer S, Denniston M. Health-related quality of life of cancer and noncancer patients in Medicare managed care. Cancer. 2003; 97:674-681. [PubMed: 12548610]

37. Tomich P, Helgeson V. Is finding something good in the bad always good? Benefit finding among women with breast cancer. Health Psychol. 2004; 23:16-23. [PubMed: 14756599]

38. Stanton, A.; Bower, J.; Low, C. Posttraumatic growth after cancer. In: Calhoun, L.; Tedeschi, R., editors. Handbook of posttraumatic growth: research and practice. Mahwah, NJ: Erlbaum; 2006. p. 138-175.

39. Zebrack B. Cancer survivors and quality of life: a critical review of the literature. Oncol Nurs Forum. 2000; 27:1395-1401. [PubMed: 11058971]

40. Elphee E. Understanding the concept of uncertainty in survivors with indolent lymphoma. Oncol Nurs Forum. 2008; 35:449-454. [PubMed: 18467294]

41. Mols F, Aaronson N, Vingerhoets A, et al. Quality of life among long-term non-Hodgkin lymphoma survivors. Cancer. 2007; 109:1659-1667. [PubMed: 17330853]

42. Bailey D, Mishel M, Belyea M. Uncertainty intervention for watchful waiting in prostate cancer. Cancer Nurs. 2004; 27:339-346. [PubMed: 15525860]

43. Pettengell R, Donatti C, Hoskin P. The impact of follicular lymphoma on health-related quality of life. Ann Oncol. 2008; 19:570-576. [PubMed: 18056649]

44. Persson L, Hallberg I. Lived experience of survivors of leukemia or malignant lymphoma. Cancer Nurs. 2004; 27:303-313. [PubMed: 15292726]

45. Arora N, Hamilton A, Potosky A, et al. Population-based survivorship research using cancer registries: a study of non-Hodgkin's lymphoma survivors. J Cancer Surviv. 2007; 1:49-63. [PubMed: 18648945]

46. Kouroukis T, Meyer R, Benger A, et al. An evaluation of age-related differences in quality of life preferences in patients with non-Hodgkin's lymphoma. Leuk Lymphoma. 2004; 45:2471-2476. [PubMed: 15621762]

47. Aaronson N, Meyerowitz B, Bard M. Quality of life research in oncology. Past achievements and future priorities. Cancer. 1991; 67:839-843. [PubMed: 1986855]

48. Chlebowski R, Chen Z, Anderson G, et al. Ethnicity and breast cancer: factors influencing differences in incidence and outcome. J Natl Cancer Inst. 2005; 97:439-448. [PubMed: 15770008] 
49. Ahles T, Saykin A, Furstenberg C, et al. Quality of life of long-term survivors of breast cancer and lymphoma treated with standard-dose chemotherapy or local therapy. J Clin Oncol. 2005; 23:4399-4405. [PubMed: 15994149]

50. Arden-Close E, Pacey A, Eiser C. Health related quality of life in survivors of lymphoma: a systematic review and methodological critique. Leuk Lymphoma. 2010; 51:628-640. [PubMed: 20218810]

51. Crespi C, Smith S, Petersen L, et al. Measuring the impact of cancer: a comparison of nonHodgkin lymphoma and breast cancer survivors. J Cancer Surviv. 2010; 4:45-58. [PubMed: 19967410]

52. Ware J, Sherbourne C. The MOS 36-item short-form health survey (SF-36). Conceptual framework and item selection. Med Care. 1992; 30:473-483. [PubMed: 1593914]

53. Cella D, Webster K, Cashy J, et al. Development of a measure of health-related quality of life for non-Hodgkin's lymphoma clinical research: the Functional Assessment of Cancer TherapyLymphoma (FACT-Lym). Blood. 2005; 106(Suppl 1):Abstract 750.

54. Cella D. The Functional Assessment of Cancer Therapy-Anemia (FACT-An) scale: a new tool for the assessment of outcomes in cancer anemia and fatigue. Semin Hematol. 1997; 34(Suppl 2):1319. [PubMed: 9253779]

55. Ferrell B, Dow K, Leigh S, et al. Quality of life in long-term cancer survivors. Oncol Nurs Forum. 1995; 22:915-922. [PubMed: 7567610]

56. Ferrell B, Dow K, Grant M. Measurement of the quality of life in cancer survivors. Qual Life Res. 1995; 4:523-531. [PubMed: 8556012]

57. Crespi C, Ganz P, Petersen L, Castillo A, Caan B. Refinement and psychometric evaluation of the Impact of Cancer Scale. J Natl Cancer Inst. 2008; 100:1530-1541. [PubMed: 18957678] 


\section{Algorithm of Article Selection Method}

Search Date: April 2010

\section{Keyword Search Results}

Medline $(n=42)$, CINAHL $(n=143)$, PsycINFO

$(n=12)$, Cochrane Library $(n=0)$

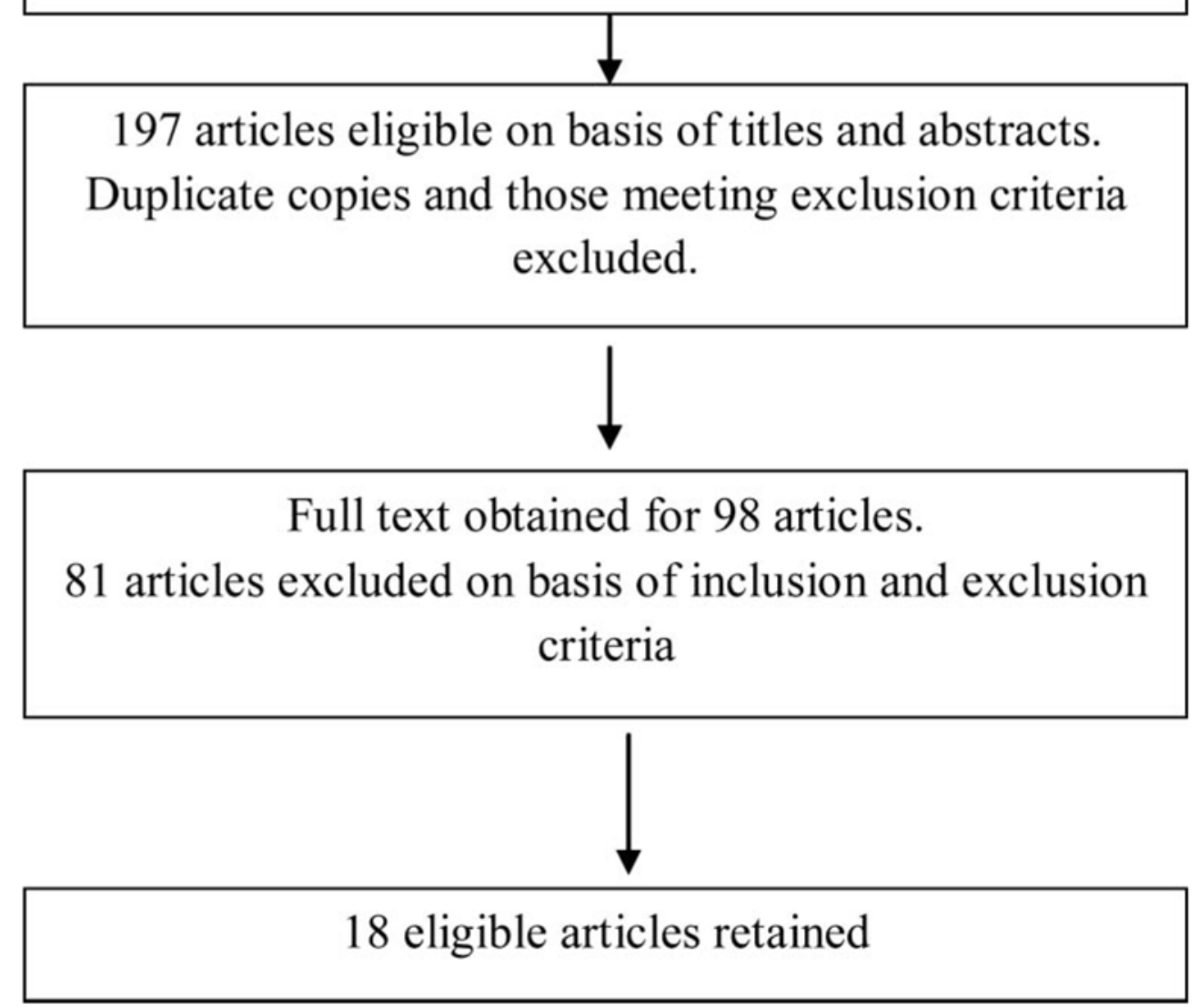

Figure 1.

Algorithm of the process used to search for and select appropriate articles to review for indicators of the quality of life of non-Hodgkin lymphoma survivors. CINAHL, Cumulative Index to Nursing and Allied Health Literature. 


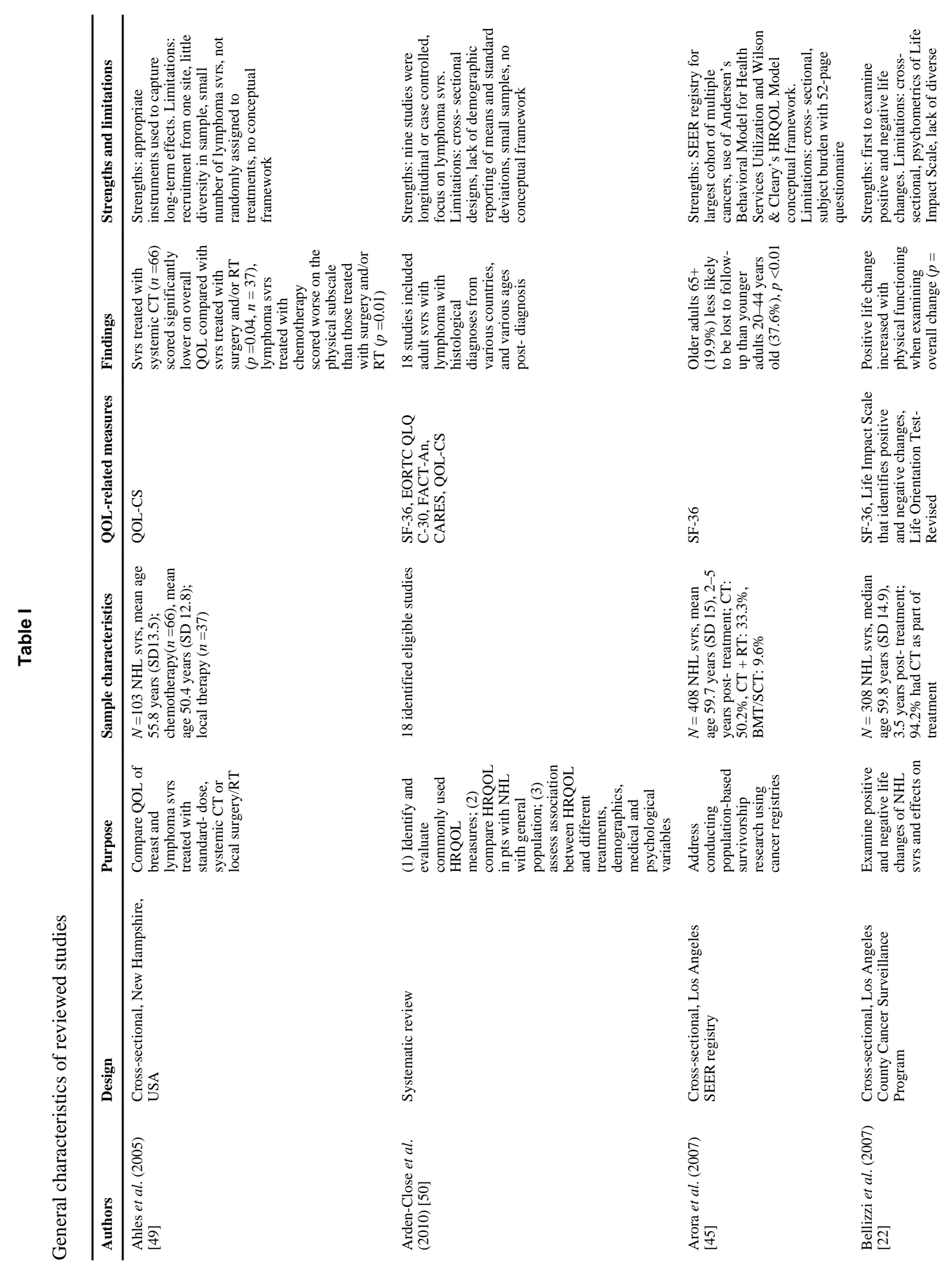

Leuk Lymphoma. Author manuscript; available in PMC 2011 August 24. 


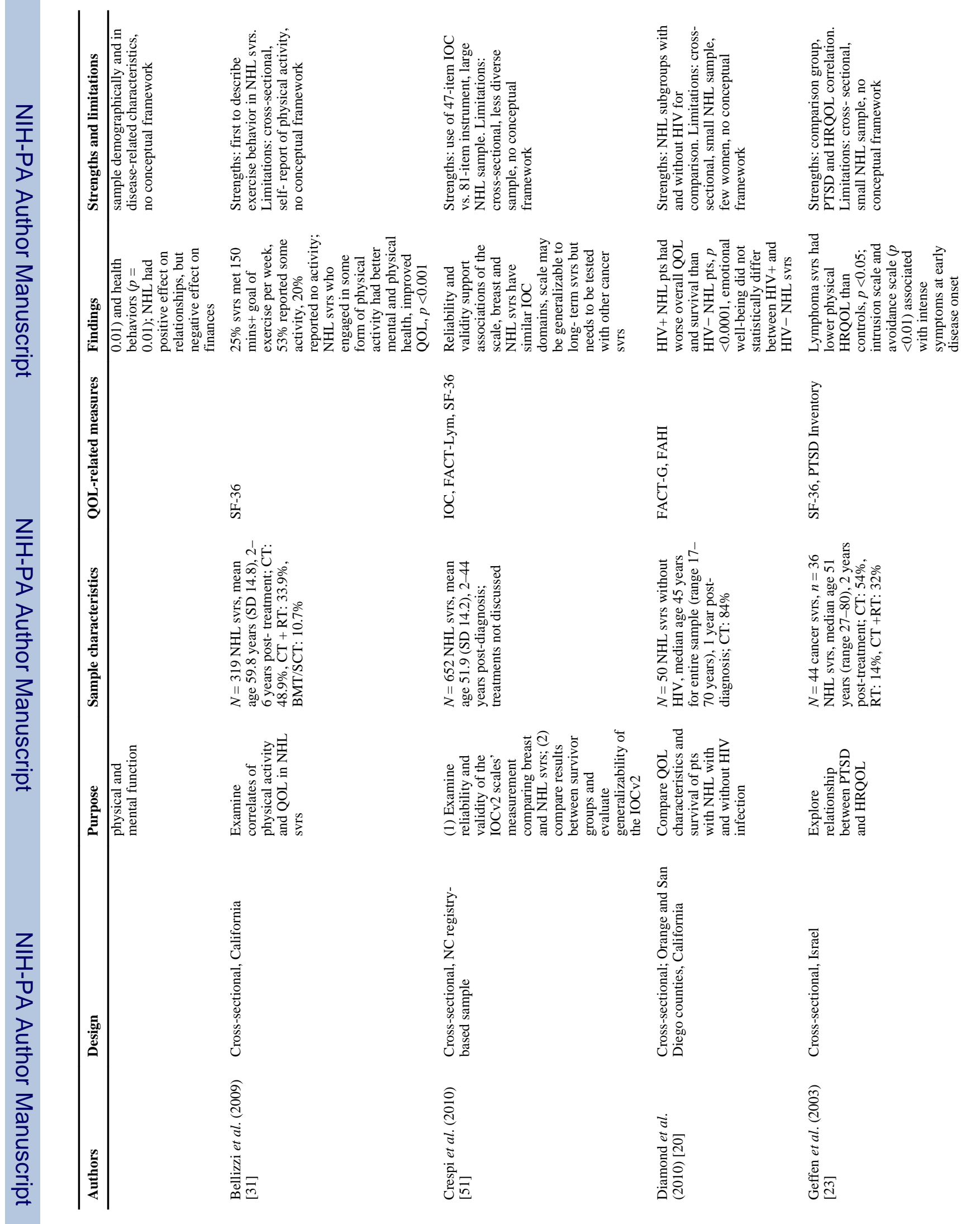



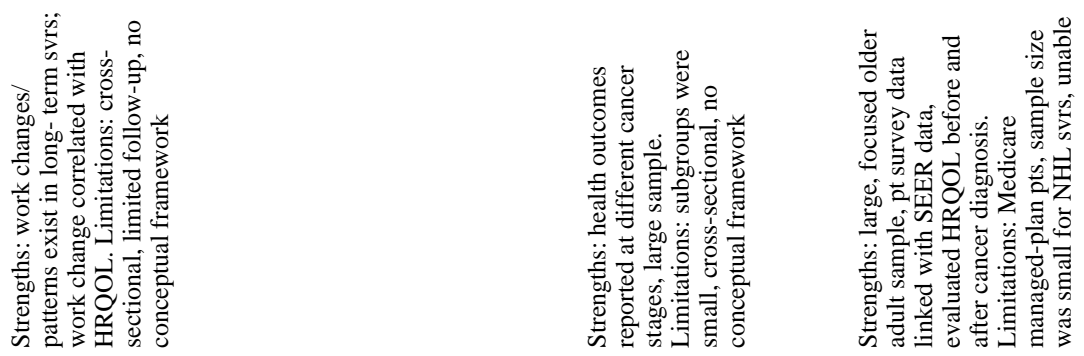

\section{$\overrightarrow{0}$}

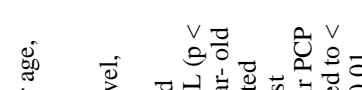

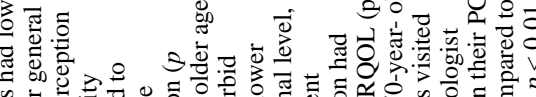

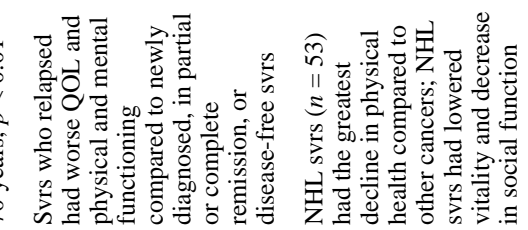

$\underset{\substack{1 \\ \text { it }}}{\infty}$

㺼

$\underset{\substack{2 \\ \text { it }}}{\infty}$

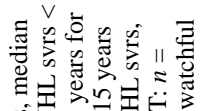

国路园管

s.

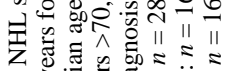

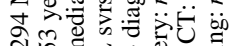

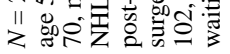

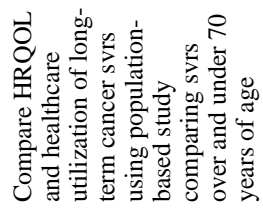

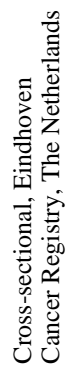

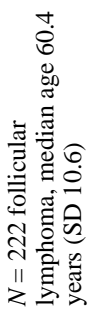
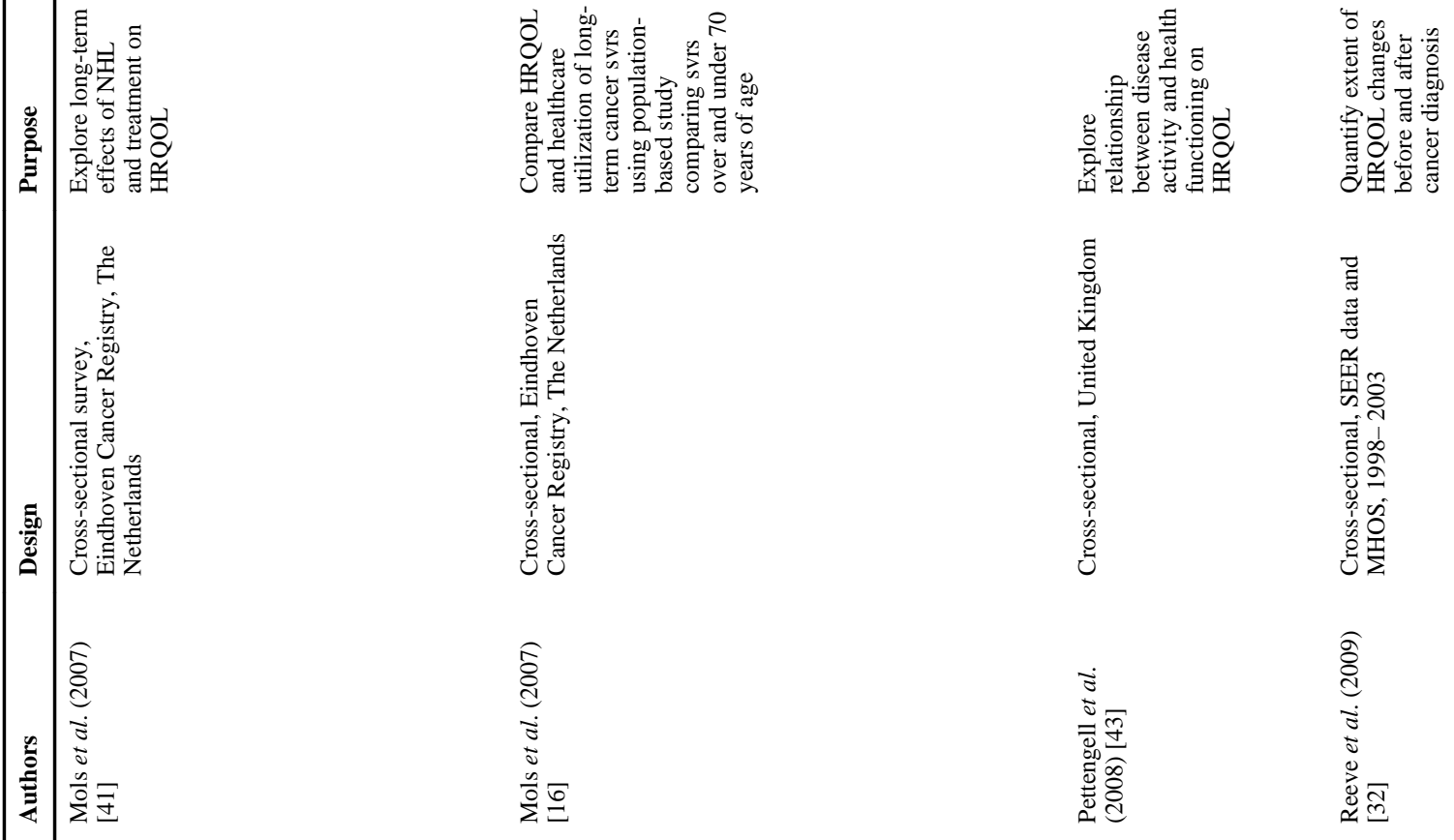

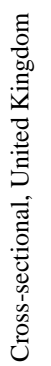

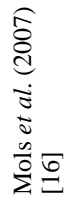

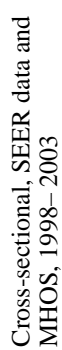

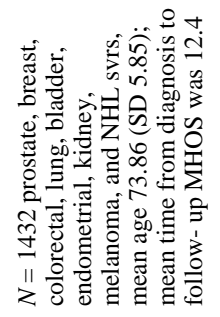

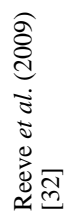



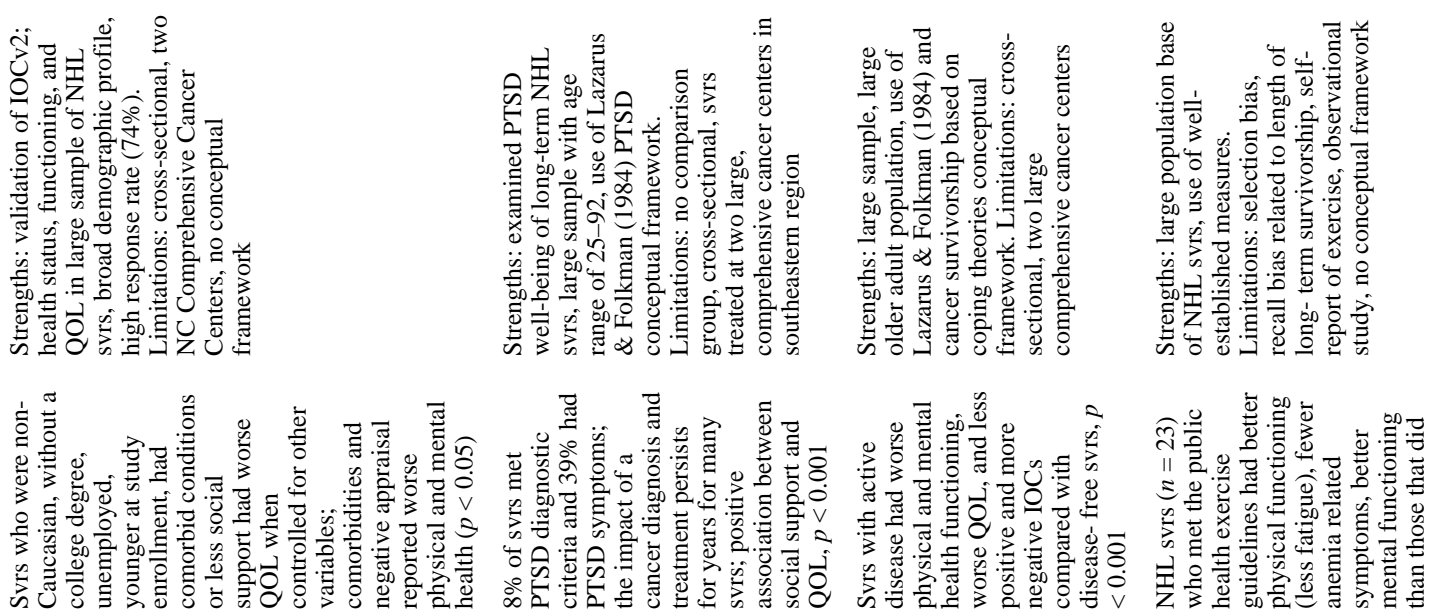

0
$\dot{0}$
$\mathbb{1}$
0
0
0
1
0
0
0
0

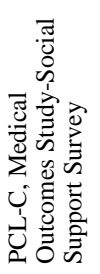

O

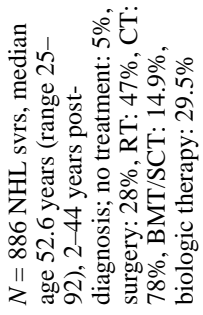

焉

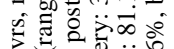

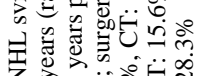

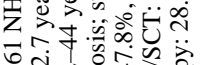

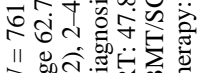

站

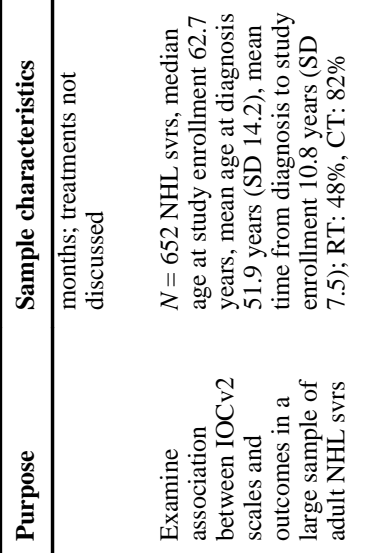

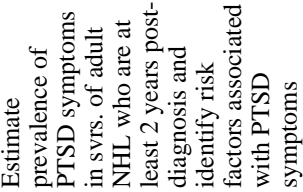

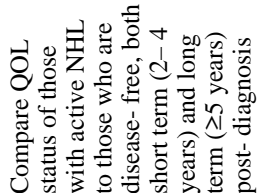

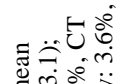

m。ำ

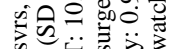

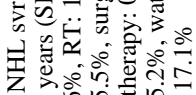

के

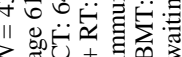

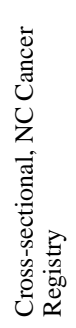
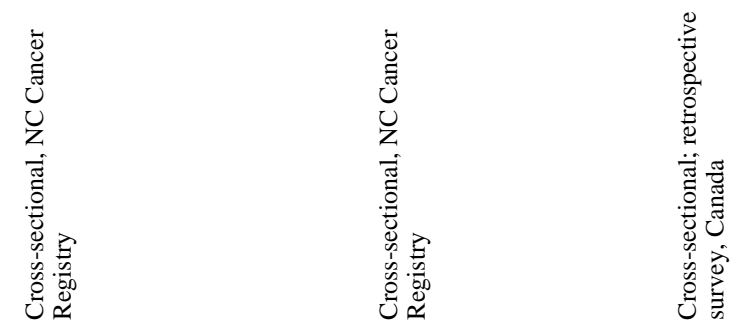

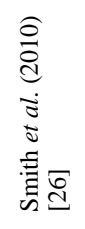

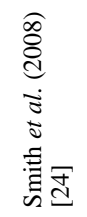

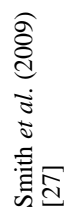

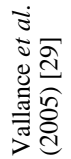




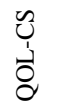

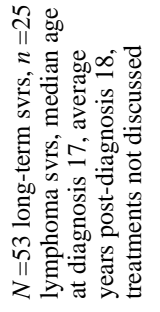

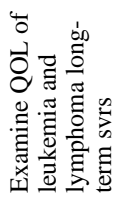

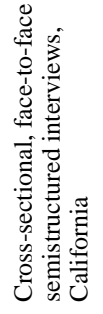

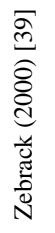

0
1
0
0
0
0
0
0
0
0
0
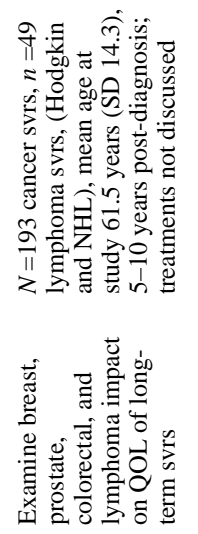

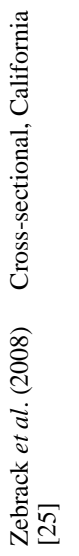

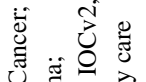

tै

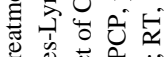

월

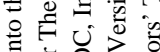

至

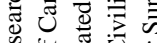

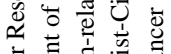

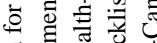

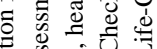

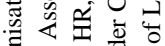

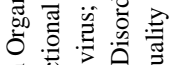

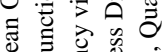

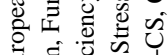

昰 है

记完

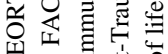

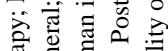

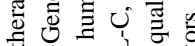

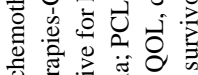

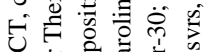

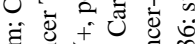

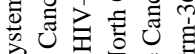

की

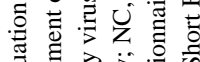

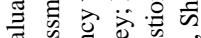

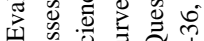

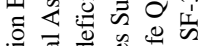

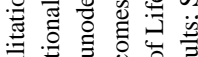

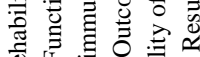

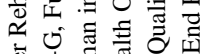

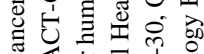

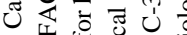

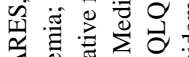

उ

较舟

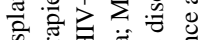

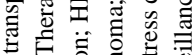

항

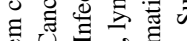

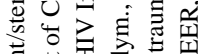

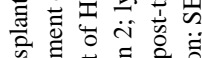

ह

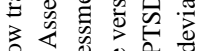

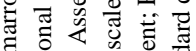

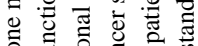

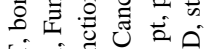

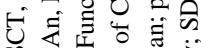

造证。

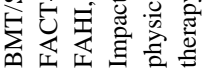




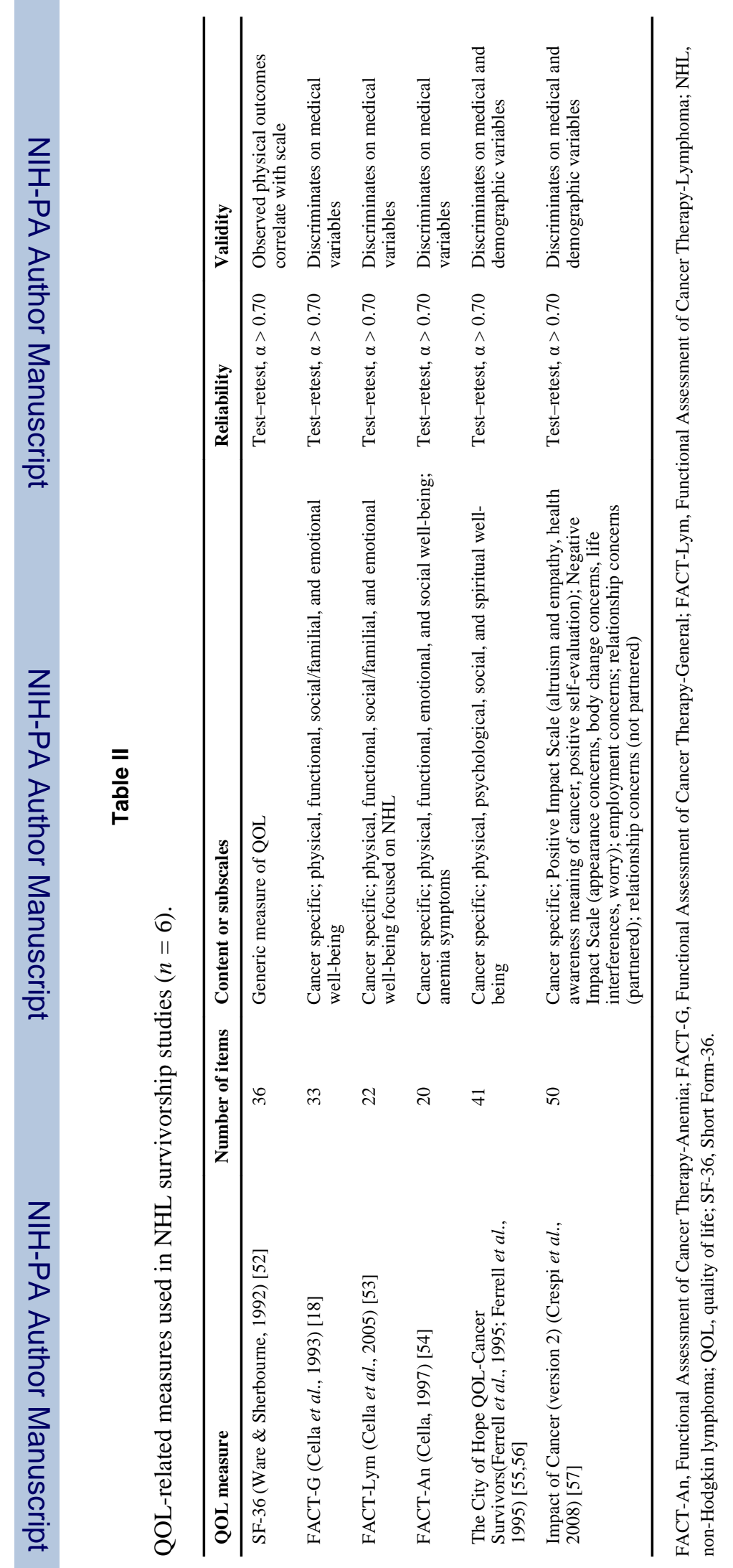

Leuk Lymphoma. Author manuscript; available in PMC 2011 August 24. 